\title{
Hi Mass Function from HIPASS
}

\author{
V. Kilborn ${ }^{1}$, R. L. Webster ${ }^{1}$ and L. Staveley-Smith ${ }^{2}$ \\ ${ }^{1}$ School of Physics, University of Melbourne, Parkville, Vic. 3052, Australia \\ vkilborn@physics.unimelb.edu.au, rwebster@physics.unimelb.edu.au \\ 2 Australia Telescope National Facility, CSIRO, PO Box 76, Epping, NSW 2121, Australia \\ Lister.Staveley-Smith@atnf.csiro.au \\ Received 1998 November 17, accepted 1999 January 28
}

\begin{abstract}
The Hi Parkes All Sky Survey (HIPASS) is a blind search for extragalactic neutral hydrogen, covering the whole of the southern sky. We present the latest $\mathrm{H}_{\mathrm{I}}$ mass function (HIMF) constructed from a sample of 263 galaxies with declinations $<-62^{\circ}$. Standard $\sum 1 / V_{\max }$ and maximum likelihood techniques are used in the analysis. No upturn in the low-mass end of the HIMF is yet seen, though our selection procedure presently conspires against the lowest-mass galaxies.
\end{abstract}

Keywords: galaxies: mass function — radio lines: galaxies — surveys

\section{Introduction}

The neutral hydrogen (HI) mass function (HIMF) describes the number density of neutral hydrogen as a function of $\mathrm{HI}$ mass at the present epoch. Measuring the HIMF is important for understanding the evolution of galaxies and for understanding the incidence of low-redshift Ly- $\alpha$ damped and 'forest' lines against background ultraviolet sources. Until recently, the data used to determine the HIMF have come from very small samples of Hi-selected objects (e.g. Zwaan et al. 1997; Schneider, Spitzak \& Rosenberg 1998), or from Hi observations of opticallyselected galaxies (e.g. Solanes, Giovanelli \& Haynes 1996). This means that HIMFs typically have poor statistics in the extreme mass bins, or are inherently biased by the optical nature of the sample selection. Optical samples tend to miss low surface-brightness systems (e.g. Chengalur, Giovanelli \& Haynes 1995) and tidal gas (e.g. Schneider et al. 1983). However, an extensive blind Hi survey such as HIPASS gives both a large and an unbiased sample and will increase the number counts for the low and high mass ends of the HIMF. Presently, the low-mass end of the HIMF is somewhat controversial with contrary views being expressed as to the relative importance of low-mass and high-mass galaxies to the overall Hi density of the Universe.

There is some evidence for an upturn at the low-luminosity end of the optical luminosity function (e.g. Loveday 1997). It is important to establish whether a similar upturn exists at the low-mass end of the HIMF. Most derivations of the HIMF have found a shallow faint-end slope (Schechter parameter, $\alpha \approx 1 \cdot 2$ - e.g. Zwaan et al. 1997), implying that low-mass galaxies contribute relatively little to the total Hi density. Recently Schneider, Spitzak \& Rosenberg (1998) claimed to see an upturn below masses of $\sim 10^{8} M_{\odot}$. However, their result is from a sample with only two galaxies in the lowest mass bin, so it remains to be seen whether this will persist in statistically larger samples.

In this paper, we derive the HIMF for a sample of $263 \mathrm{HI}$-selected galaxies. In Section 2, we give the particulars of the HIPASS survey and the data selection process; in Section 3 we derive the HIMF. In Section 4, we briefly discuss the results. A value of $H_{0}=100 \mathrm{hm} \mathrm{s}^{-1} \mathrm{Mpc}^{-1}$ is used throughout.

\section{Survey Parameters and Data Selection}

HIPASS is being conducted at the $64 \mathrm{~m}$ Parkes Radio Telescope in NSW, Australia. The survey uses the multibeam receiver, which has individual beam sizes on the sky of $14^{\prime}$. The sky is actively scanned in $3^{\circ} \times 8^{\circ}$ strips, and it will be scanned five times to reach the final survey sensitivity. HIPASS will survey the southern sky between $-1200 \mathrm{~km} \mathrm{~s}^{-1}$ and $12700 \mathrm{~km} \mathrm{~s}^{-1}$, with a velocity resolution of 18 $\mathrm{km} \mathrm{s}^{-1}$. The RMS noise in the final data cubes is $\sim 13$ mJy per beam. Bandpass subtraction is undertaken in real-time at the telescope (Barnes et al. 1998), and the data are gridded at a later stage into $8^{\circ} \times 8^{\circ}$ cubes, with a pixel size of $4^{\prime} \times 4^{\prime}$ and velocity spacing of $13 \cdot 2 \mathrm{~km} \mathrm{~s}^{-1}$. There will be 388 data cubes in the finished southern sky survey. The $3 \sigma \mathrm{HI}$ mass limit for the survey is $\sim 1.4 \times 10^{6} D_{\mathrm{Mpc}}^{2} M_{\odot}$, and the $3 \sigma$ Hi column density limit is $\sim 7 \times 10^{17} \mathrm{~cm}^{-2}$ per velocity channel.

A follow-up observation program is taking place at the Australia Telescope Compact Array (ATCA) to obtain better positional accuracy of interesting sources, and optical follow-up is undertaken at the Siding Spring and CTIO 40 inch telescopes. These observations are vital to the survey, as they will confirm marginal detections and provide greater 


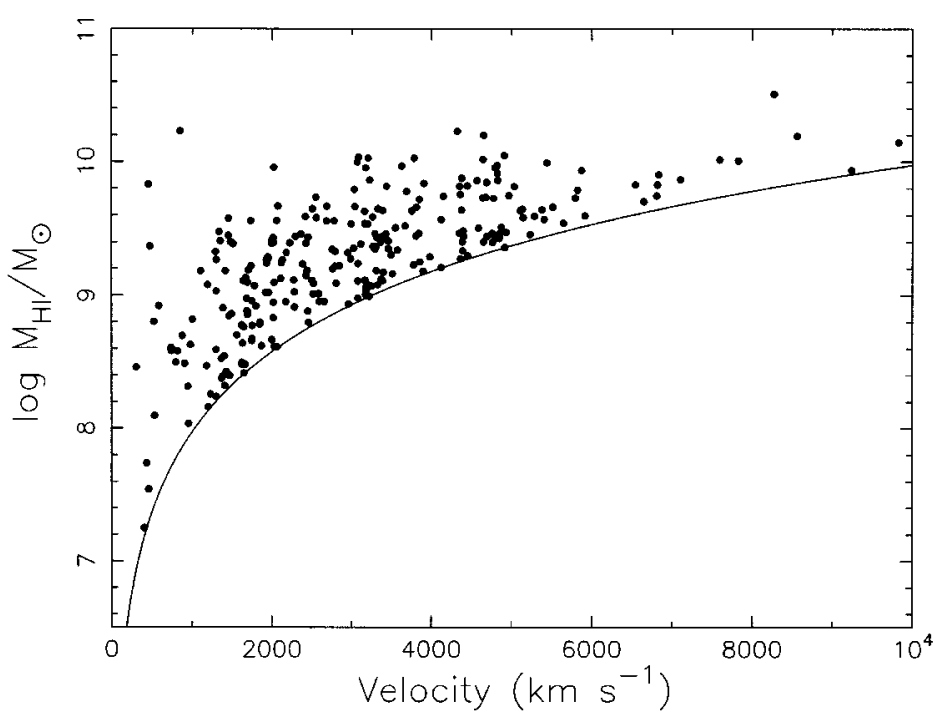

Figure 1-Mass of the galaxies in the sample versus velocity. The curve represents the theoretical mass cut-off for the sample.

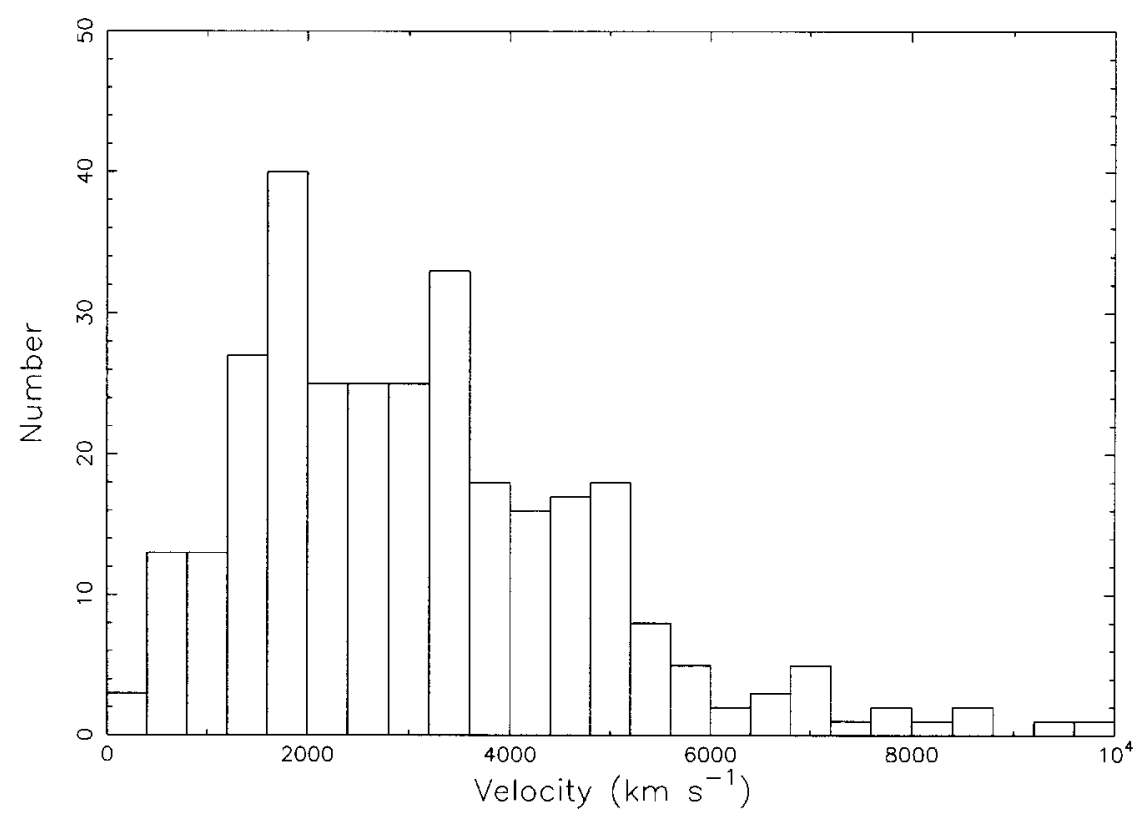

Figure 2-Velocity distribution for the full sample.

resolution for interesting objects such as intergalactic Hi clouds.

We have selected our sample of galaxies from the declination region $-62^{\circ}>\delta>-70^{\circ}$ and $\delta<-78^{\circ}$, through the full range in Right Ascension. This region has been scanned to the full multibeam sensitivity of five scans, and represents $0.572 \mathrm{sr}$ in area $(7 \%$ of the total HIPASS survey area). The galaxies were detected by eye in the data cubes; in total, 304 galaxies were found in this region. Of the sample, $15 \%$ are previously uncatalogued and $30 \%$ are new detections in Hi. There are no confirmed detections of Hi clouds without optical counterparts in this sample.

To construct a complete sample, a cut-off was made at the integrated flux value of $4 \mathrm{Jy} \mathrm{km} \mathrm{s}^{-1}$.
This was chosen as a conservative limit, because the detection limits for the HIPASS data have not been explored fully at this stage. By choosing such a conservative cut-off, the detections with the lowest peak flux and velocity width are omitted from the sample, and we are limited to characterising the HIMF above a mass of $M_{\mathrm{HI}} \sim 10^{7} M_{\odot}$. This means that once again the low mass end of the HIMF will be determined on the data from just a couple of galaxies. A plot showing the theoretical mass cut-off versus the masses in the sample is shown in Figure 1, which shows that the sample seems to be complete to the chosen cut-off. With this integrated flux limit imposed, there were 263 galaxies left in the sample. A histogram showing the velocity distribution of the sample can be seen 


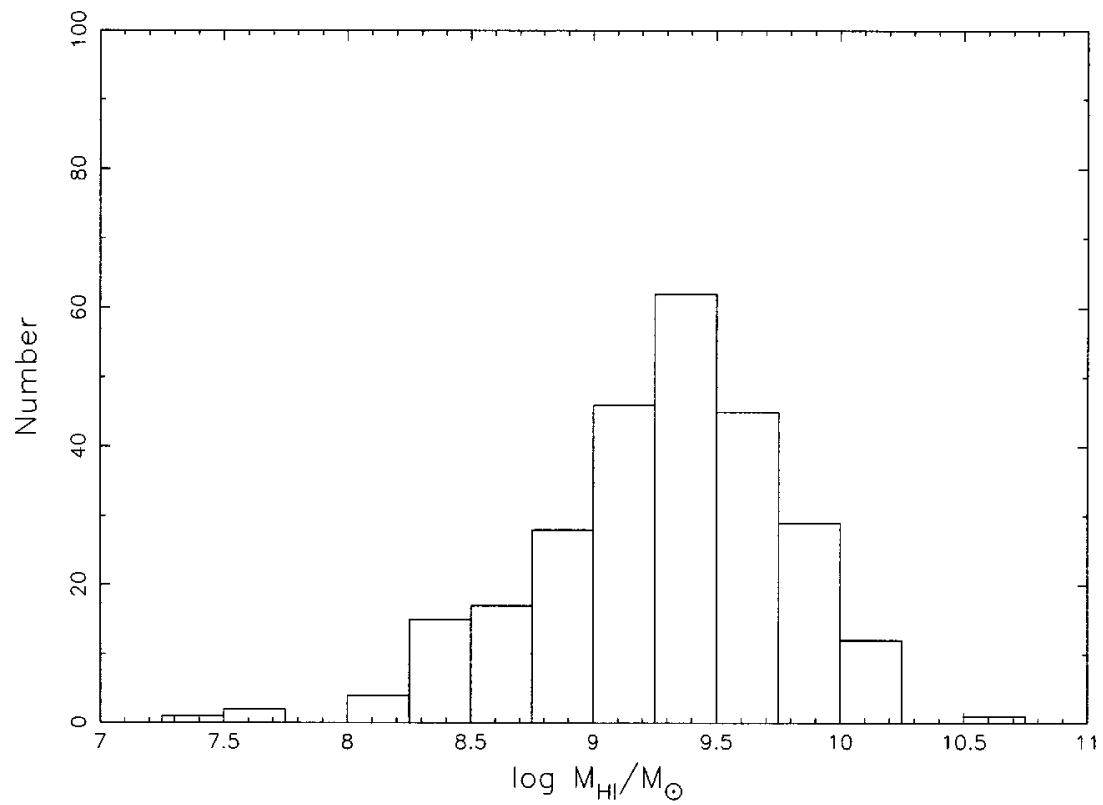

Figure 3-Mass distribution for the flux limited sample.

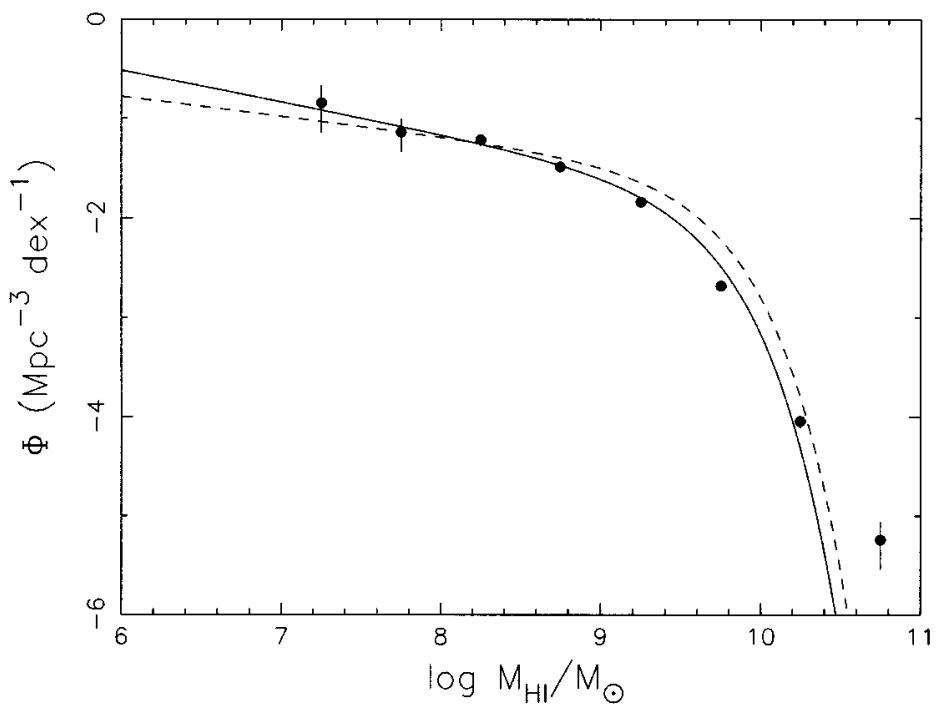

Figure 4-Hi mass function (HIMF): the solid points represent the measured $\mathrm{HI}$ mass function per decade, and the solid line represents the best fit Schechter function for the $\sum 1 / V_{\max }$ method from this sample: $\alpha=1 \cdot 32, \log M^{*}=9 \cdot 5$ and $\theta^{*}=0 \cdot 01$. The dotted line is the HIMF from Zwaan et al. (1997), with $\alpha=1 \cdot 2$ and $\log M^{*}=9 \cdot 55$. The error bars show $1 \sigma$ errors (Marshall 1985).

in Figure 2-we have detected galaxies between 400 $\mathrm{km} \mathrm{s}^{-1}$ and $10,000 \mathrm{~km} \mathrm{~s}^{-1}$ in this sample. The mass distribution for the sample can be seen in Figure 3. While the number statistics have been increased in the higher mass bins, the two lowest bins combined have just three galaxies.

\section{Results}

The standard $\sum 1 / V_{\max }$ method (Schmidt 1968) was used to determine the HIMF from the data. A Schechter (1976) function was then fitted to the data:

$$
\begin{aligned}
\Phi\left(\frac{M_{\mathrm{HI}}}{M^{*}}\right) d\left(\frac{M_{\mathrm{HI}}}{M^{*}}\right) & =\theta^{*}\left(\frac{M_{\mathrm{HI}}}{M^{*}}\right)^{-\alpha} \\
\times & \exp \left(-\frac{M_{\mathrm{HI}}}{M^{*}}\right) d\left(\frac{M_{\mathrm{HI}}}{M^{*}}\right),
\end{aligned}
$$

where $M^{*}$ is the mass that defines the characteristic knee in the function, and $\theta^{*}$ is the normalisation. Figure 4 shows the HIMF derived using this method, and the best fit Schechter function is shown by the solid line. The slope of the low-mass end is $\alpha=1 \cdot 32$, the characteristic mass, $\log M^{*}=9 \cdot 5$, and the normalisation, $\theta^{*}=0 \cdot 01$. These are similar 
to values found by Zwaan et al. (1997), who find $\alpha=1 \cdot 2$, and $\log M^{*}=9 \cdot 55$ (see the dotted line in Figure 4 ). The normalisation found for the HIPASS sample is slightly lower than the Zwaan et al. value of $0 \cdot 014$. There is no indication from our data that there is a turn-up in the slope of the HIMF to the mass limit we have imposed. However, as we still have poor statistics in the low mass bins (a single galaxy in the lowest mass bin), the faint end slope is still somewhat uncertain.

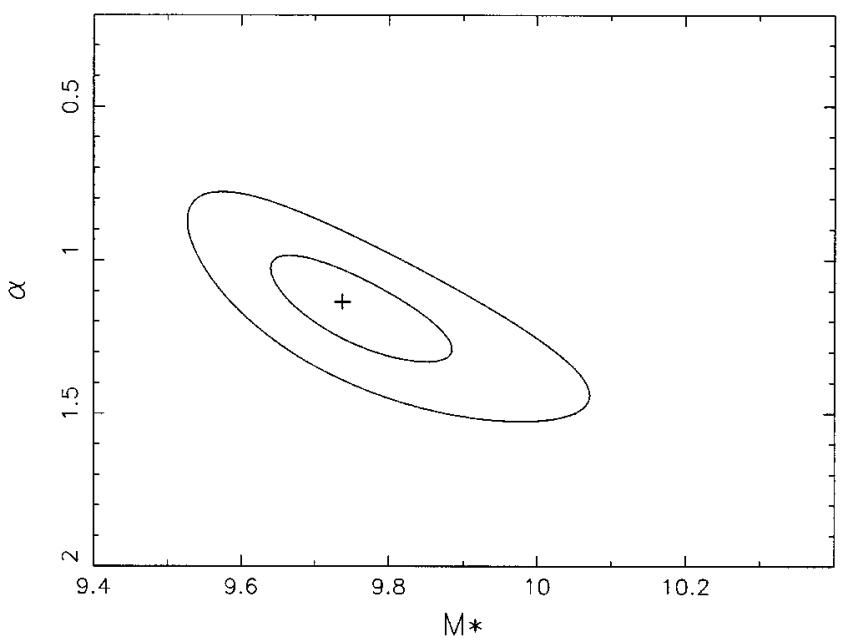

Figure 5-Contours from the maximum likelihood method. The maximum value is seen at $\alpha=1 \cdot 15, \log M^{*}=9 \cdot 74$. The contours are $68 \cdot 3 \%$ and $99 \cdot 9 \%$ confidence limits.

A maximum likelihood method (Sandage, Tammann \& Yahil 1979) was also used to determine Schechter parameters for the data. The Schechter parameters determined using this method are slightly different from the best fit parameters found using the $\sum 1 / V_{\max }$ method, with $\alpha=1 \cdot 15$ and $\log M^{*}=$ $9 \cdot 74$. Figure 5 shows a plot of contours of likelihood for the sample. It is possible that the difference in value of the $\sum 1 / V_{\max }$ method and the maximum likelihood method is due to clustering of the galaxies in this region. The maximum likelihood method is not dependent on the distribution of galaxies in the sample, whereas the $\sum 1 / V_{\max }$ varies according to the clustering of the galaxies in the sample (Binggeli, Sandage \& Tammann 1988). A full investigation of selection effects and different computation methods will be presented in a later paper.

Using the $\sum 1 / V_{\max }$ Schechter function parameters derived for this sample we can determine the $\mathrm{HI}$ density at the present epoch, which is $\rho_{\mathrm{HI}}=\Gamma(2-$ a) $M^{*} \theta^{*}$, where $\Gamma$ is the Euler gamma function. We obtain $\rho_{\mathrm{HI}}=4 \cdot 2 \times 10^{7} h M_{\odot} \mathrm{Mpc}^{-3}$, or $2 \cdot 8 \times 10^{-33} h$ $\mathrm{g} \mathrm{cm}^{-3}$. The cosmological mass density of $\mathrm{HI}$ at $z=0, \Omega_{\mathrm{HI}}$, is the ratio of the $\mathrm{HI}_{\mathrm{I}}$ density over the critical density at the present epoch ( $\rho_{c}=1.88 \times 10^{-29} h^{2} \mathrm{~g} \mathrm{~cm}^{-3}$, Padmanabhan 1993). This gives a value of $\Omega_{\mathrm{HI}}=1.5 \times 10^{-4} h^{-1}$. Assuming that the mass percentage of $\mathrm{HeI}$ is $25 \%$, the total cosmological mass density of neutral gas at the present epoch is $\Omega_{g}=1.88 \times 10^{-4} h^{-1}$ : this is slighty lower than the Zwaan et al. (1997) value of $\Omega_{g}(z=0)=(2 \cdot 7 \pm 0 \cdot 5) \times 10^{-4} h^{-1}$.

\section{Discussion and Conclusions}

So far, we have taken a conservative cut-off to derive the HIMF. This has excluded the low mass galaxies which we are particularly interested in, and in the future we will work on determining the sensitivity limits of the survey more fully so that all the available data can be included in the analysis. The preliminary results from HIPASS show an HIMF that does not indicate a steep rise at the faint end, which is a similar result to previous surveys. We derive a cosmological mass density of $\Omega_{g}=1.88 \times 10^{-4} h^{-1}$, which is also similar to past results. With better understanding of the survey sensitivity we will be able to push the limits of the low mass end of the HIMF.

\section{Acknowledgments}

We acknowledge the multibeam working group for their help with observations, and thank the Parkes Observatory staff for their constant support.

\section{References}

Barnes, D. G., et al. 1998, in ADASS VII (San Fransisco: ASP), p. 89

Binggeli, B., Sandage, A., \& Tammann, G. A. 1988, A\&A, 26,509

Chengalur, J. N., Giovanelli, R., \& Haynes, M. P. 1995, AJ, 109,2415

Loveday, J. 1997, ApJ, 489, 29

Marshall, H. 1985, ApJ, 299, 109

Padmanabhan, T. 1993, in Structure Formation in the Universe (Cambridge University Press), p. 29

Sandage, A., Tamman, G. A., \& Yahil, A. 1979, ApJ, 232, 352

Schechter, P. 1976, ApJ, 203, 297

Schmidt, M. 1968, ApJ, 151, 393

Schneider, S. E., Helou, G., Salpeter, E. E., \& Terzianm, Y. 1983, ApJ, 273L, 1

Schneider, S. E., Spitzak, J. G., \& Rosenberg, J. L. 1998, ApJ, 507, L9

Solanes, J., Giovanelli, R., \& Haynes, M. 1996, ApJ, 461, 609

Zwaan, M. A., Briggs, F. H., Sprayberry, D., \& Sorar, E. 1997, ApJ, 490, 173 